\title{
BRANCHING LAWS: SOME RESULTS AND NEW EXAMPLES
}

\author{
OSCAR MÁRQUEZ, SEBASTIÁN SIMONDI, AND JORGE A. VARGAS
}

\begin{abstract}
For a connected, noncompact simple matrix Lie group $G$ so that a maximal compact subgroup $K$ has a three dimensional simple ideal, in this note we analyze the admissibility of the restriction of irreducible square integrable representations for the ambient group when they are restricted to certain subgroups that contain the three dimensional ideal. In this setting we provide a formula for the multiplicity of the irreducible factors. Also, for general $G$ such that $G / K$ is an Hermitian $G$-manifold we give a necessary and sufficient condition so that an arbitrary square integrable representation of the ambient group is admissible over the semisimple factor of $K$.
\end{abstract}

\section{INTRODUCTION}

Let $G$ be a connected noncompact simple matrix Lie group. Henceforth, we fix a maximal compact subgroup $K$ of $G$ and we assume both groups have the same rank. We also fix $T \subset K$ a maximal torus. Thus, $T$ is a compact Cartan subgroup of $G$. Under these hypotheses, Harish-Chandra showed there exists irreducible unitary representations of $G$ so that its matrix coefficients are square integrable with respect to a Haar measure on $G$. One aim of this note is to write down explicit branching laws for the restriction of some irreducible square integrable representation to specific subgroups $H$ of $G$. A second objective is to show that when $G$ is simple, the symmetric space $G / K$ has $G$-invariant quaternionic structure, and $H$ is a specific subgroup locally isomorphic to the group $\mathrm{SU}(2,1)$, then an irreducible square integrable representation for $G$ has an admissible restriction to $H$ if and only if it is a quaternionic discrete series representation. The last objective is to present results on admissible restriction of square integrable representations to specific subgroups of $G$. To begin with, we recall a description of the irreducible square integrable representations for $G$. Harish-Chandra showed that the set of equivalence classes of irreducible square integrable representations is parameterized by a lattice contained in the dual of the Lie algebra of a compact Cartan subgroup. In order to state our results we need to specify the parametrization and set up some notation. As usual, the Lie algebra of a Lie group is denoted by the corresponding

2010 Mathematics Subject Classification. Primary 22E46; Secondary 17B10.

Key words and phrases. Square integrable representation; Admissible restriction; Multiplicity formulae.

Partially supported by CONICET and SECYT-UNC, Argentina. 
lower case German letter. The complexification of a real vector space $V$ is denoted by adding the subindex $\mathbb{C}$. However, the root space for a root is denoted by the real Lie algebra followed by a subindex equal to the root. $V^{\star}$ denotes the dual space to a vector space $V$. Let $\theta$ be the Cartan involution which corresponds to the subgroup $K$, the associated Cartan decomposition is denoted by $\mathfrak{g}=\mathfrak{k}+\mathfrak{p}$. Let $\Phi(\mathfrak{g}, \mathfrak{t})$ denote the root system attached to the Cartan subalgebra $\mathfrak{t}_{\mathbb{C}}$. Hence, $\Phi(\mathfrak{g}, \mathfrak{t})=\Phi_{c} \cup \Phi_{n}=\Phi(\mathfrak{k}, \mathfrak{t}) \cup \Phi_{n}(\mathfrak{g}, \mathfrak{t})$ splits up as the disjoint union of the set of compact roots and the set of noncompact roots. From now on, we fix a system of positive roots $\Delta$ for $\Phi_{c}$. For this note, either the highest weight or the infinitesimal character of an irreducible representation of $K$ is dominant with respect to $\Delta$. The Killing form on the Lie algebra $\mathfrak{g}$ gives rise to an inner product $($,$) in i \mathfrak{t}^{\star}$. As usual, let $\rho=\rho_{g}$ denote half of the sum of the roots for some system of positive roots for $\Phi(\mathfrak{g}, \mathfrak{t})$.

A Harish-Chandra parameter for $G$ is $\lambda \in i t^{\star} \operatorname{such}$ that $(\lambda, \alpha) \neq 0$ for every $\alpha \in \Phi(\mathfrak{g}, \mathfrak{t})$, and so that $\lambda+\rho$ is the differential of a character of $T$. To each HarishChandra parameter, $\lambda$, Harish-Chandra associated a unique irreducible square integrable representation $\left(\pi_{\lambda}^{G}, V_{\lambda}\right)$ of $G$. Moreover, he showed the map $\lambda \mapsto \pi_{\lambda}^{G}$ is a bijection from the set of Harish-Chandra parameters dominant with respect to $\Delta$ onto the set of equivalence classes of irreducible square integrable representations for $G$. For a proof see [12].

In [2, Gross and Wallach have considered a quaternionic real form $G$ of a complex simple Lie group and constructed a specific subgroup $H$ locally isomorphic to $\mathrm{SU}(2,1)$; their setting is as follows: a system of positive roots $\Psi$ so that $\Delta \subset \Psi \subset \Phi(\mathfrak{g}, \mathfrak{t})$ is called small if the maximal root $\beta$ for $\Psi$ is compact, $\Psi$ has at most two noncompact simple roots $\alpha_{0}, \alpha_{1}$ and after we write $\beta=n_{0} \alpha_{0}+n_{1} \alpha_{1}+$ a linear combination of compact simple roots, we have the inequality $n_{0}+n_{1} \leq 2$. A noncompact connected simple Lie group $G$ is a quaternionic real form if $\mathfrak{g}$ is an inner form of a complex simple Lie algebra and if a compactly imbedded Cartan subalgebra $\mathfrak{t}$ has the property that $\Phi(\mathfrak{g}, \mathfrak{t})$ admits a small system of positive roots so that $n_{0}+n_{1}=2$. In [2], the list of the Lie algebras for the quaternionic real forms is presented; we reproduce the list in section 3. It can be shown that the set of equivalence classes of the set of quaternionic real forms is equal to the set of equivalence classes of the set of noncompact simple Lie groups $G$ so that the associated global symmetric space admits a $G$-invariant quaternionic structure.

In order to state Theorem 1, we fix a quaternionic real form $G$, a small system of positive roots $\Psi \supset \Delta$, and a noncompact simple root $\alpha$ for $\Psi$. An irreducible square integrable representation $\left(\pi_{\lambda}^{G}, V\right)$ is called quaternionic discrete series representation if the Harish-Chandra parameter $\lambda$ is dominant with respect to $\Psi$.

For the quaternionic real form $G$, a particular copy $\mathfrak{h}$ of $\mathfrak{s u}(2,1)$ contained in $\mathfrak{g}$ is constructed in [2]. For this, Gross and Wallach verify the equality

$$
\frac{2(\beta, \alpha)}{(\alpha, \alpha)}=1
$$

Thus, the Lie subalgebra $\mathfrak{h}_{\mathbb{C}}$ of $\mathfrak{g}_{\mathbb{C}}$ spanned by the root vectors corresponding to the roots $\{ \pm \alpha, \pm \beta\}$ is isomorphic to $\mathfrak{s l}(3, \mathbb{C})$ and invariant under the conjugation 
of $\mathfrak{g}_{\mathbb{C}}$ with respect to $\mathfrak{g}$. Hence, $\mathfrak{h}:=\mathfrak{g} \cap \mathfrak{h}_{\mathbb{C}}$ is a real form for $\mathfrak{h}_{\mathbb{C}}$. This real form has a compactly embedded Cartan subalgebra, namely, $\mathfrak{u}:=\mathfrak{t} \cap \mathfrak{h}$. Thus, $\mathfrak{h}$ is isomorphic to $\mathfrak{s u}(2,1)$. Henceforth, we identify the set $\Phi(\mathfrak{h}, \mathfrak{u})$ with the subset $\{ \pm \alpha, \pm \beta, \pm(\beta-\alpha)\}$ of $\Phi(\mathfrak{g}, \mathfrak{t})$.

(1.0) Let $H$ denote the analytic subgroup of $G$ with Lie algebra $\mathfrak{h}$. Then, $L:=$ $K \cap H$ is a maximal compact subgroup for $H$. The system $\Phi(\mathfrak{h}, \mathfrak{u})$ has three systems of positive roots to which the root $\beta$ belongs to. The one that interests us is the non-holomorphic system

$$
\Psi_{q}:=\Psi \cap \Phi(\mathfrak{h}, \mathfrak{u})=\{\beta-\alpha, \alpha, \beta\} .
$$

The simple roots for $\Psi_{q}$ are $\beta-\alpha, \alpha$. For a root $\gamma \in \Phi(\mathfrak{g}, \mathfrak{t})$, we denote its coroot by $\check{\gamma} \in i$ t. Let $\Lambda_{1}, \Lambda_{2}$ denote the fundamental weights for $\Psi_{q}$, labeled so that $\Lambda_{1}(\check{\alpha})=0$.

(1.1) Owing to results in [1, 13, 7], which we will review in section 2, it follows that for a Harish-Chandra parameter $\lambda$ dominant with respect to the small system $\Psi$ the irreducible representation $\left(\pi_{\lambda}^{G}, V_{\lambda}\right)$ restricted to $H$ is an admissible representation. That is, there exists a sequence of Harish-Chandra parameters for $H$, dominant with respect to $\beta$,

$$
\mu_{1}, \mu_{2}, \ldots, \mu_{j}, \ldots \text { in } i \mathfrak{u}^{\star}
$$

and there exist positive integers

$$
n^{G, H}\left(\lambda, \mu_{j}\right), \quad j=1,2, \ldots
$$

so that the restriction of $\left(\pi_{\lambda}^{G}, V_{\lambda}\right)$ to $H$ is unitarily equivalent to the discrete Hilbert sum

$$
\sum_{j=1}^{\infty} n^{G, H}\left(\lambda, \mu_{j}\right)\left(\pi_{\mu_{j}}^{H}, V_{\mu_{j}}\right) .
$$

In [2] it is shown that $\Psi_{n}:=\Psi \cap \Phi_{n}$ has $2 d$ elements. Our hypothesis that $G$ is a quaternionic real form forces the root spaces for the roots $\pm \beta$ span a three dimensional simple ideal $\mathfrak{s u}_{2}(\beta)$ in $\mathfrak{k}$. We denote by $\mathfrak{k}_{2}$ the complementary ideal to $\mathfrak{s u}_{2}(\beta)$ in $\mathfrak{k}$. Hence, we have the decompositions

$$
\mathfrak{t}=\mathbb{R} i \check{\beta}+\left(\mathfrak{t} \cap \mathfrak{k}_{2}\right) \quad \text { and } \quad \Delta=\{\beta\} \cup \Phi\left(\mathfrak{k}_{2}, \mathfrak{t} \cap \mathfrak{k}_{2}\right) \cap \Psi .
$$

For each $\lambda \in \mathfrak{t}_{\mathbb{C}}$, we write $\lambda=\lambda_{1}+\lambda_{2}$, with $\lambda_{1} \in \mathbb{C} \check{\beta}, \lambda_{2} \in \mathfrak{t}_{2_{\mathbb{C}}}:=\mathfrak{t}_{\mathbb{C}} \cap \mathfrak{k}_{\mathbb{C}_{\mathbb{C}}}$. Let $q_{\mathfrak{u}}: \mathfrak{t}^{\star} \rightarrow \mathfrak{u}^{\star}$ denote the restriction map.

(1.2) We will verify, in (2.7), that for a Harish-Chandra parameter $\lambda$ dominant for the small system $\Psi$, we have that $\lambda_{2}$ is a Harish-Chandra parameter for $K_{2}$ or perhaps for a two fold cover of $K_{2}$. From now on, $\pi_{\lambda_{2}}^{K_{2}}$ denotes the irreducible representation for $\mathfrak{k}_{2}$ of infinitesimal character $\lambda_{2}$. As usual, $\Delta_{T \cap K_{2}}\left(\pi_{\lambda_{2}}^{K_{2}}\right)$ denotes the set of $T \cap K_{2}$-weights for the representation $\pi_{\lambda_{2}}^{K_{2}}$ and $M\left(\lambda_{2}, \nu\right)$ stands for the multiplicity of the weight $\nu \in \Delta_{T \cap K_{2}}\left(\pi_{\lambda_{2}}^{K_{2}}\right)$.

In (2.7) we verify that for $\lambda$ dominant with respect to the small system $\Psi$ the weight $\lambda_{1}+\nu+a \Lambda_{1}+b q_{\mathfrak{u}}\left(\lambda_{2}\right)$ is dominant with respect to the system $\Psi_{q}$ for every 
$a, b \in \mathbb{Z}_{\geq 0}$, and for every $U \cap K_{2}$-weight $\nu$ of $\pi_{\lambda_{2}}^{K_{2}}$. One result of this note is the next theorem.

Theorem 1. Let $G$ be a quaternionic real form, $H$ as in (1.0) and $\left(\pi_{\lambda}^{G}, V_{\lambda}\right)$ a quaternionic discrete series representation for $G$. Then, $n^{G, H}(\lambda, \mu) \neq 0$ if and only if $\mu=(n+d) \Lambda_{1}+(m+d) \Lambda_{2}+q_{\mathfrak{u}}\left(\lambda_{2}\right)+q_{\mathfrak{u}}(\nu)$ with arbitrary $m, n \in \mathbb{Z}_{\geq 0}$ and $T \cap K_{2}$-weight $\nu$ for $\pi_{\lambda_{2}}^{K_{2}}$. Moreover,

$$
\begin{aligned}
& n^{G, H}(\lambda, \mu)= \\
& \sum_{\substack{\nu \in \Delta_{T \cap K_{2}}\left(\pi_{\lambda_{2}}^{K_{2}}\right), m, n \in \mathbb{Z}_{\geq 0},}} M\left(\lambda_{2}, \nu\right)\left(\begin{array}{c}
m+d-2 \\
d-2
\end{array}\right)\left(\begin{array}{c}
n+d-2 \\
d-2
\end{array}\right) .
\end{aligned}
$$

A question that naturally arises is: What are the Harish-Chandra parameters for $G$, dominant with respect to $\Delta$, so that $\pi_{\lambda}^{G}$ has an admissible restriction to $H$ ? The answer to this question is given in Proposition 1

A group $G$ locally isomorphic to either $\mathrm{SO}(3, n)$ shares with the quaternionic real forms that a suitable copy of the algebra $\mathfrak{s u}_{2}$ is an ideal in a maximal compactly embedded subalgebra for $\mathfrak{g}$. A group locally isomorphic to $\mathrm{SO}(3,2 p+1)$ has no square integrable representations. For a group locally isomorphic to $\mathrm{SO}(3,2 n)$ and $n \geq 2$, in Proposition 2 we show that no irreducible square integrable representation of $G$ has an admissible restriction to the usual copy of "SO$(3)$ " contained in $G$. For the quaternionic group $\operatorname{Sp}(1, p)$ the usual factor " $\operatorname{Sp}(1)$ " of a maximal compact subgroup is contained in a certain image $H_{0}$ of $\mathrm{Sp}(1,1)$. In Proposition 3 we show that a quaternionic discrete series for $S p(p, 1)$ has admissible restriction to $H_{0}$. Additionally, we compute the Harish-Chandra parameter of each irreducible $H_{0^{-}}$ factor as well as the multiplicity of each factor.

The group $\mathrm{SU}(2,1)$ can be mapped into a simple Lie group $G$ in perhaps several ways by maps $\phi: \mathrm{SU}(2,1) \rightarrow G$. A natural question is: What is the triple $\left(G, \pi_{\lambda}^{G}, \phi\right)$ such that $\pi_{\lambda}$ restricted to the image of $\phi$ is an admissible representation? In [10] we find that for the analytic subgroup $H_{1}$ that corresponds to the image of $\mathfrak{s u}(2,1)$ in the rank one real form of a complex group type $F_{4}$ no square integrable representation of the ambient group has an admissible restriction to $H_{1}$.

We would like to comment that this note grew up from results in the $\mathrm{Ph}$. D. theses of Sebastián Simondi and Oscar Márquez, successfully defended at the Faculty of Mathematics, Astronomy and Physics at the Universidad Nacional de Córdoba, Argentina, in 2007 and 2011 respectively.

\section{Proof of Theorem 1}

As in the hypothesis $G$ is a connected, quaternionic simple Lie group and $H$ is the subgroup locally isomorphic to $\mathrm{SU}(2,1)$. To begin with, we sketch a proof for the statement: For $\lambda$ dominant with respect to the small system $\Psi$, the representation $\left(\pi_{\lambda}^{G}, V_{\lambda}\right)$ restricted to $H$ is admissible. In fact, for a system of positive roots $\Sigma \subset$ $\Phi(\mathfrak{g}, \mathfrak{t})$ in [1] is attached an ideal $\mathfrak{k}_{1}(\Sigma)$ for the Lie algebra $\mathfrak{k}$. The ideal is equal 
to the real form of the ideal of $\mathfrak{k}_{\mathbb{C}}$ spanned by $\left\{\left[Y_{\gamma}, Y_{\phi}\right]: \gamma, \phi \in \Sigma \cap \Phi_{n}, Y_{\gamma} \in g_{\gamma}\right\}$ together with a subspace of the center $\mathfrak{z} \mathfrak{k}$ of $\mathfrak{k}$.

(2.0) For the system $\Psi$, cf. [2, Prop. 1.3, Table 2.5], we have that any root in $\Phi_{c} \cap \Psi$ not equal to $\beta$ is a linear combination of compact simple roots for $\Psi$. Thus, for two noncompact roots in $\Psi$, their sum is a root only when the sum is equal to $\beta$. Thus, $\mathfrak{k}_{1}(\Psi)$ is equal to $\mathfrak{s u}_{2}(\beta)$ plus the contribution of the center. Now, from the list of the quaternionic real forms, we read that $\mathfrak{z k}$ is nonzero only for $G$ locally isomorphic to $\mathrm{SU}(2, p)$. For $\mathfrak{s u}(2, p)$, it is shown in [1] that for $\Psi$ the contribution of $\mathfrak{z}_{\mathfrak{k}}$ to $\mathfrak{k}_{1}(\Psi)$ is just the zero subspace. Hence, for a quaternionic system $\Psi$ we have

$$
\mathfrak{k}_{1}(\Psi)=\mathfrak{s u}_{2}(\beta) .
$$

Because of the definition of $H$ we have that $K_{1}(\Psi)$ is contained in $H$, hence Theorem 1 in [1] yields that for $\lambda$ dominant with respect to $\Psi$ the representation $\left(\pi_{\lambda}^{G}, V_{\lambda}\right)$ has an admissible restriction to $H \cap K$ as well as to the subgroup $H$. In [6] we find a different proof of the admissibility.

Therefore, there exists a sequence of Harish-Chandra parameters for $H, \mu_{1}, \ldots$, $\mu_{n}, \ldots \in i \mathfrak{u}^{\star}$, for which we may assume for every $j,\left(\mu_{j}, \beta\right)>0$, and positive integers $n^{G, H}\left(\lambda, \mu_{j}\right)$ so that the restriction of $\left(\pi_{\lambda}^{G}, V_{\lambda}\right)$ restricted to $H$ is equivalent to the Hilbert sum

$$
\sum_{j} n^{G, H}\left(\lambda, \mu_{j}\right) \pi_{\mu_{j}}^{H}
$$

We are left to compute $\mu_{j}$, to show that each $\mu_{j}$ is dominant for $\Psi_{q}$, and to compute the integers $n^{G, H}\left(\lambda, \mu_{j}\right)$.

For this we recall results in [1, 4. For $\gamma \in i \mathbf{t}^{\star}$ (resp. in $\left.i \mathfrak{u}^{\star}\right)$ we consider the Dirac distribution $\delta_{\gamma}$ and the discrete Heaviside distribution defined by the series

$$
y_{\gamma}:=\sum_{n \geq 0} \delta_{\frac{\gamma}{2}+n \gamma}=\delta_{\frac{\gamma}{2}}+\delta_{\frac{\gamma}{2}+\gamma}+\delta_{\frac{\gamma}{2}+2 \gamma}+\ldots
$$

For any strict multiset $\gamma_{1}, \ldots, \gamma_{r}$ the convolution $y_{\gamma_{1}} \star \cdots \star y_{\gamma_{r}}$ is a well defined distribution. In particular, we have

$$
\underbrace{y_{\gamma} \star \cdots \star y_{\gamma}}_{r}:=y_{\gamma}^{r}=\sum_{n \geq 0}\left(\begin{array}{c}
n+r-1 \\
r-1
\end{array}\right) \delta_{\left(\frac{r}{2}+n\right) \gamma} .
$$

We have

$$
\mathfrak{u}_{\mathbb{C}}=\mathbb{C} \check{\alpha}+\mathbb{C} \check{\beta}, \quad \mathfrak{u}_{\mathbb{C}} \cap \mathfrak{k}_{2_{\mathbb{C}}}=\mathfrak{u}_{\mathbb{C}} \cap \mathfrak{t}_{2_{\mathbb{C}}}=\mathfrak{h}_{\mathbb{C}} \cap \mathfrak{k}_{2_{\mathbb{C}}}=\mathbb{C}(\check{\beta}-2 \check{\alpha})=\mathbb{C} \Lambda_{2} .
$$

$q_{\mathfrak{u}}: \mathfrak{t}_{\mathbb{C}}^{\star} \rightarrow \mathfrak{u}_{\mathbb{C}}^{\star}$ denotes restriction map.

Next, we recall the sub-root system $\Phi_{\mathfrak{z}}:=\left\{\gamma \in \Phi(\mathfrak{k}, \mathfrak{t}): q_{\mathfrak{u}}(\gamma)=0\right\}$. Because of (1.0) and (1.1),

$$
\Phi_{\mathfrak{z}}=\{\gamma:(\gamma, \alpha)=(\gamma, \beta)=0\}=\left\{\gamma \in \Phi\left(\mathfrak{k}_{2}, \mathfrak{t}_{2}\right): q_{\mathfrak{u} \cap \mathfrak{t}_{2}}(\gamma)=0\right\} .
$$

The Weyl group for the system $\Phi_{\mathfrak{z}}$ is denoted by $W_{\mathfrak{z}}$. Because of $(2.0)$ the Weyl group $W$ for the pair $(\mathfrak{k}, \mathfrak{t})$ is equal to the product $\left\langle S_{\beta}\right\rangle \times W\left(\mathfrak{k}_{2}, \mathfrak{t}\right)$. Thus,

$$
W_{\mathfrak{z}} \backslash W=\left\langle S_{\beta}\right\rangle \times W_{\mathfrak{z}} \backslash W\left(\mathfrak{k}_{2}, \mathfrak{t}\right) .
$$


Let

$$
\begin{aligned}
\Delta(\mathfrak{k} / \mathfrak{l}) & :=q_{\mathfrak{u}}\left[\Psi \cap \Phi(\mathfrak{k}, \mathfrak{t}) \backslash \Phi_{\mathfrak{z}}\right] \backslash \Phi(\mathfrak{l}, \mathfrak{u})=q_{\mathfrak{u}}\left[\{\beta\} \cup \Psi\left(\mathfrak{k}_{2}, \mathfrak{t}\right) \backslash \Phi_{\mathfrak{z}}\right] \backslash\{\beta\} \\
& =q_{\mathfrak{u} \cap \mathfrak{k}_{2}}\left(\Psi \cap \Phi\left(\mathfrak{k}_{2}, t \cap \mathfrak{k}_{2}\right) \backslash \Phi_{\mathfrak{z}}\right)=: \Delta\left(\mathfrak{k}_{2} / \mathfrak{u} \cap \mathfrak{k}_{2}\right) .
\end{aligned}
$$

We set $\rho_{\mathfrak{z}}=\frac{1}{2} \sum_{\gamma \in \Psi \cap \Phi_{\mathfrak{z}}} \gamma$, and for $\sigma \in i \mathbf{t}^{\star}$ the Weyl polynomial is defined to be

$$
\varpi(\sigma):=\frac{\prod_{\gamma \in \Psi \cap \Phi_{\mathfrak{z}}}(\sigma, \gamma)}{\prod_{\gamma \in \Psi \cap \Phi_{\mathfrak{z}}}\left(\rho_{\mathfrak{z}}, \gamma\right)} .
$$

As before, we write $\lambda=\lambda_{1}+\lambda_{2}$, with $\lambda_{1} \in \mathbb{R} i \check{\beta}$ and $\lambda_{2} \in \mathfrak{t}_{2}$. Then, owing to (1.2), for $\gamma \in \Phi\left(\mathfrak{k}_{2}, \mathfrak{t}\right) \cap \Psi$ we have the equality $\lambda(\check{\gamma})=\lambda_{2}(\check{\gamma})$. Thus, $\lambda_{2}$ is a HarishChandra parameter for $K_{2}$ or perhaps for a two-fold cover of $K_{2}$. Actually, it readily follows that $\lambda_{2}$ is a Harish-Chandra parameter for $K_{2}$ if and only if $\frac{\beta}{2}$ lifts to a character of $T$. Therefore, replacing if necessary $G$ by a two-fold cover, we have that $\lambda_{2}$ is a Harish-Chandra parameter for $K_{2}$.

We now state according to [4] the branching law for the restriction of the irreducible representation $\pi_{\lambda_{2}}^{K_{2}}$ of infinitesimal character $\lambda_{2}$ to the one dimensional torus $H \cap K_{2}=U \cap K_{2}$. The restriction of $\pi_{\gamma}^{K_{2}}$ to $H \cap K_{2}$ is the sum of onedimensional representations $\sigma_{1}, \ldots, \sigma_{r}$ with multiplicity $M\left(\lambda_{2}, \sigma_{j}\right)$ for $j=1, \ldots, r$. The formula of Heckman for this particular case reads

$$
\begin{aligned}
\sum_{\mu \in \Delta_{U \cap K_{2}}\left(\pi_{\lambda_{2}}^{K_{2}}\right)} M\left(\lambda_{2}, \mu\right) \delta_{\mu} & \\
= & \sum_{s \in W_{\mathfrak{z}} \backslash W\left(\mathfrak{k}_{2}, \mathfrak{t}\right)} \epsilon(s) \varpi\left(s \lambda_{2}\right) \delta_{q_{\mathfrak{u} \cap \mathfrak{k}_{2}}\left(s \lambda_{2}\right)} \star y_{\Delta\left(\mathfrak{k}_{2} / \mathfrak{u} \cap \mathfrak{k}_{2}\right)} .
\end{aligned}
$$

Another fact needed for the proof is a formula in [1] for the restriction of $\pi_{\lambda}^{G}$ to the subgroup $H$. The hypothesis for the truth of the formula is $K_{1}(\Psi)$ being a subgroup of $H$, which in our case holds because of our choice of $\Psi$ and $H$. The hypothesis on $G$ and on the system $\Psi$ yields that for each $w \in W$ the multiset

$$
S_{w}^{H}:=\left[\Delta(\mathfrak{k} / \mathfrak{l}) \cup q_{\mathfrak{u}}\left(w \Psi_{n}\right)\right] \backslash \Phi(\mathfrak{h}, \mathfrak{u})
$$

is strict. This also follows from an explicit computation of $S_{w}^{H}$, which we will carry out later on. The formula that encodes the parameters $\mu_{j}$ and the multiplicities $n^{G, H}\left(\lambda, \mu_{j}\right)$ is:

$$
\sum_{\mu \in i \mathfrak{u}^{\star}:(\mu, \beta)>0} n^{G, H}(\lambda, \mu)\left(\delta_{\mu}-\delta_{S_{\beta} \mu}\right)=\sum_{w \in W_{\mathfrak{\jmath}} \backslash W} \epsilon(w) \varpi(w \lambda) \delta_{q_{\mathfrak{u}}(w \lambda) \star y_{S_{w}^{H}}}
$$

To elaborate on 2.4 and 2.5 we recall a few known results. It is convenient to think of $\left(\mathfrak{u} \cap \mathfrak{s u}_{2}(\beta)\right)^{\star}$ (resp. $\left.\mathfrak{t}_{2}^{\star}\right)$ as the linear functionals on $\mathfrak{t}$ that vanish on $\mathfrak{t}_{2}$ (resp. on $\mathfrak{u} \cap \mathfrak{s u}_{2}(\beta)$ ); hence, for $\lambda_{2} \in \mathfrak{t}_{2}$, we have the equality $q_{\mathfrak{u}}\left(\lambda_{2}\right)=q_{\mathfrak{u} \cap \mathfrak{k}_{2}}\left(\lambda_{2}\right)$. 
For $w \in W\left(\mathfrak{k}_{2}, \mathfrak{t}_{2}\right)$ we have the equalities

$$
\begin{aligned}
q_{\mathfrak{u}}(w \lambda) & =\lambda_{1}+q_{{\mathfrak{u} \cap \mathfrak{k}_{2}}\left(w \lambda_{2}\right)} \\
q_{\mathfrak{u}}\left(w S_{\beta} \lambda\right) & =S_{\beta}\left(\lambda_{1}\right)+q_{\mathfrak{u \cap k}_{2}}\left(w \lambda_{2}\right) \\
\varpi(w \lambda) & =\varpi\left(w \lambda_{2}\right) \\
\varpi\left(w S_{\beta} \lambda\right) & =\varpi\left(w \lambda_{2}\right) .
\end{aligned}
$$

From Table 2.5 in [2] it follows that any root in $\Phi\left(\mathfrak{k}_{2}, \mathfrak{t}\right)$ is a linear combination of compact simple roots in $\Psi$. Thus, Lemma 3.3 in [3] yields

$$
w \Psi_{n}=\Psi_{n} \quad \text { for } w \in W\left(\mathfrak{k}_{2}, \mathfrak{t}\right) .
$$

In [2, Proposition 1.3] it is shown that $\Psi_{n}=\left\{\gamma \in \Psi: \frac{2(\beta, \gamma)}{(\beta, \beta)}=1\right\}$, and that the map $\gamma \mapsto \beta-\gamma$ is an involution in $\Psi_{n}$. Thus, the number of elements of $\Psi_{n}$ is an even number $2 d$ and we may write

$$
\Psi_{n}=\left\{\gamma_{2}, \ldots, \gamma_{d}, \beta-\gamma_{2}, \ldots, \beta-\gamma_{d}, \alpha, \beta-\alpha\right\} .
$$

Hence, we have $S_{\beta}\left(\Psi_{n}\right)=-\Psi_{n}$. Also in [2, Proposition 2] it is shown that

$$
q_{\mathfrak{u}}\left(\gamma_{j}\right)=\Lambda_{1} \quad \text { for } j=2, \ldots, d \text {. }
$$

The equality $\Lambda_{1}+\Lambda_{2}=\beta$ yields $q_{\mathfrak{u}}\left(\beta-\gamma_{j}\right)=\Lambda_{2}$. From these and 2.6 we conclude that, for $w \in W\left(\mathfrak{k}_{2}, \mathfrak{t}\right)$,

$$
\begin{gathered}
q_{\mathfrak{u}}\left(w \Psi_{n}\right)=\{\underbrace{\Lambda_{1}, \ldots, \Lambda_{1}}_{d-1}, \underbrace{\Lambda_{2}, \ldots, \Lambda_{2}}_{d-1}, \alpha, \beta-\alpha\}, \\
q_{\mathfrak{u}}\left(w S_{\beta} \Psi_{n}\right)=S_{\beta}\left(q_{\mathfrak{u}}\left(w \Psi_{n}\right)\right)=\{\underbrace{\Lambda_{1}, \ldots, \Lambda_{1}}_{d-1}, \underbrace{\Lambda_{2}, \ldots, \Lambda_{2}}_{d-1}, \alpha, \alpha-\beta\} .
\end{gathered}
$$

The previous calculations let us conclude that, for $w \in W\left(\mathfrak{k}_{2}, \mathfrak{t}_{2}\right)$,

$$
\begin{aligned}
S_{w}^{H} & =\{\underbrace{\Lambda_{1}, \ldots, \Lambda_{1}}_{d-1}, \underbrace{\Lambda_{2}, \ldots, \Lambda_{2}}_{d-1}\} \cup \Delta\left(\mathfrak{k}_{2} / \mathfrak{u} \cap \mathfrak{k}_{2}\right) \\
S_{S_{\beta} w}^{H} & =\{\underbrace{-\Lambda_{1}, \ldots,-\Lambda_{1}}_{d-1}, \underbrace{-\Lambda_{2}, \ldots,-\Lambda_{2}}_{d-1}\} \cup \Delta\left(\mathfrak{k}_{2} / \mathfrak{u} \cap \mathfrak{k}_{2}\right) .
\end{aligned}
$$

The right hand side of (2.5), after we apply the previous calculations, becomes equal to

$$
\begin{aligned}
& \sum_{s \in W_{\mathfrak{z}} \backslash W\left(\mathfrak{k}_{2}\right)} \epsilon(s) \varpi\left(s \lambda_{2}\right) \delta_{\lambda_{1}} \star \delta_{q_{\mathfrak{u \cap} \mathfrak{k}_{2}}}\left(s \lambda_{2}\right) \star y_{\Lambda_{1}}^{d-1} \star y_{\Lambda_{2}}^{d-1} \star y_{\Delta\left(\mathfrak{k}_{2} / \mathfrak{u} \cap \mathfrak{k}_{2}\right)} \\
& \quad-\sum_{s \in W_{\mathfrak{\jmath}} \backslash W\left(\mathfrak{k}_{2}\right)} \epsilon(s) \varpi\left(s \lambda_{2}\right) \delta_{S_{\beta} \lambda_{1}} \star \delta_{q_{\mathfrak{u n k}_{2}}}\left(s \lambda_{2}\right) \star y_{S_{\beta} \Lambda_{1}}^{d-1} \star y_{S_{\beta} \Lambda_{2}}^{d-1} \star y_{\Delta\left(\mathfrak{k}_{2} / \mathfrak{u} \cap \mathfrak{k}_{2}\right)} \\
& =\sum_{\sigma \in \Delta_{U \cap K_{2}}\left(\pi_{\lambda_{2}}^{K_{2}}\right)} M\left(\lambda_{2}, \sigma\right) \delta_{\sigma} \star\left[\delta_{\lambda_{1}} \star y_{\Lambda_{1}}^{d-1} \star y_{\Lambda_{2}}^{d-1} \star \delta_{S \beta \lambda_{1}} \star y_{S_{\beta} \Lambda_{1}}^{d-1} \star y_{S_{\beta} \Lambda_{2}}^{d-1}\right]
\end{aligned}
$$




$$
\begin{aligned}
& =\sum_{\sigma, p, q \in \mathbb{Z}_{\geq 0}} M\left(\lambda_{2}, \sigma\right)\left(\begin{array}{c}
p+d-2 \\
d-2
\end{array}\right)\left(\begin{array}{c}
q+d-2 \\
d-2
\end{array}\right) \delta_{\lambda_{1}+\sigma} \star \delta_{p \Lambda_{1}} \star \delta_{q \Lambda_{2}} \\
& \quad+\sum_{\sigma, p, q \in \mathbb{Z}_{\geq 0}} M\left(\lambda_{2}, \sigma\right)\left(\begin{array}{c}
p+d-2 \\
d-2
\end{array}\right)\left(\begin{array}{c}
q+d-2 \\
d-2
\end{array}\right) \delta_{S_{\beta}\left(\lambda_{1}+\sigma\right)} \star \delta_{p S_{\beta} \Lambda_{1}} \star \delta_{q S_{\beta} \Lambda_{2}} .
\end{aligned}
$$

(2.7) We now show: For every $p, q \in \mathbb{Z}_{\geq 0}$, and for every $U \cap K_{2}$-weight $\sigma$ of $\pi_{\lambda_{2}}^{K_{2}}$ the weight $\lambda_{1}+\sigma+p \Lambda_{1}+q q_{\mathfrak{u}}\left(\lambda_{2}\right)$ is dominant with respect to the system $\Psi_{q}=\{\alpha, \beta-\alpha, \beta\}$.

In fact, because of a theorem of Kostant, every $T_{2}$-weight of $\pi_{\lambda_{2}}^{K_{2}}$ lies in the convex hull of $\left\{s\left(\lambda_{2}\right), s \in W\left(\mathfrak{k}_{2}, \mathfrak{t}\right)\right\}$. Thus, there exist non negative real numbers $c_{t}$ so that $\sigma=\sum_{t \in W\left(\mathfrak{k}_{2}, \mathfrak{t}\right)} c_{t} q_{\mathfrak{u}}\left(t \lambda_{2}\right)$ and $\sum_{t} c_{t}=1$. The hypothesis that $\lambda$ is regular and dominant with respect to $\Psi$ yields $\lambda_{1}(\check{\beta})=\lambda(\check{\beta})>0$.

We write $\left(\lambda_{1}+\sigma+p \Lambda_{1}+q \lambda_{2}, \alpha\right)=\lambda(\check{\beta}) \alpha(\check{\beta})+\sum_{t}\left(q_{\mathfrak{u}}\left(t \lambda_{2}\right), \alpha\right)+p\left(\Lambda_{1}, \alpha\right)$ and $q_{\mathfrak{u}}\left(t \lambda_{2}\right)=\left(t \lambda_{2}, \beta-2 \alpha\right)(\beta-2 \alpha)$. Now, since $\alpha \in i \mathfrak{u}^{\star}$, we have

$$
\left(q_{\mathfrak{u}}\left(t \lambda_{2}\right), \alpha\right)=\left(t \lambda_{2}, \alpha\right)=\left(\lambda_{2}, t^{-1} \alpha\right)=\left(\lambda, t^{-1} \alpha\right)>0,
$$

because $t$ is a product of reflections about compact simple roots for $\Psi, \alpha \in \Psi_{n}$ and (2.7).

For

$$
\begin{aligned}
\left(\lambda_{1}+\sigma+p \Lambda_{1}+\right. & \left.q \lambda_{2}, \beta-\alpha\right) \\
& =\sum_{t} c_{t}\left(t \lambda_{1}, \beta-\alpha\right)+\sum_{t} c_{t}\left(t \lambda_{2}, \beta-\alpha\right)+q\left(\lambda_{2}, \beta-\alpha\right) \\
& =\sum_{t} c_{t}\left(q_{\mathfrak{u}}\left(t \lambda_{1}+t \lambda_{2}\right), \beta-\alpha\right)+q\left(\lambda_{2}, \beta-\alpha\right) \\
& =\sum_{t} c_{t}(t \lambda, \beta-\alpha)>0,
\end{aligned}
$$

because of $\beta-\alpha \in \Psi_{n}, t \in W\left(\mathfrak{k}_{2}, \mathfrak{t}\right)$ and $\lambda$ is regular dominant for $\Psi$. We have concluded the proof of Theorem 1 , because we have shown that the left hand side of (2.5) is expressed as claims the statement of Theorem 1 This finishes the proof of Theorem 1

Note. Wallach in [13] considered the case where the lowest $K$-type for $\pi_{\lambda}$ is equal to a representation of $\mathfrak{s u}_{2}(\beta)$ times the trivial representation of $K_{2}$.

\section{Admissible Restrictions to "SU(2,1)" of Discrete Series for QUATERNIONIC REAL FORMS}

To begin with we list the Lie algebra of the Lie groups where Theorem 1 applies. Up to equivalence, the list of the Lie algebras for quaternionic real forms is: $\mathfrak{s u}(2, n)$, $\mathfrak{s o}(4, n), \mathrm{EII}=\mathfrak{e}_{6(2)}, \mathrm{EVI}=\mathfrak{e}_{7(-5)}, \mathrm{EIX}=\mathfrak{e}_{8(-24)}, \mathrm{FI}=\mathfrak{f}_{4(4)}$ and $\mathrm{G}=\mathfrak{g}_{2(2)}$. 
For the corresponding groups, we show that a square integrable irreducible representation for $G$ has an admissible restriction to the subgroup $H$ locally isomorphic to $\mathrm{SU}(2,1)$ constructed by Gross-Wallach if and only if the Harish-Chandra parameter is dominant with respect to the small system $\Psi$.

Proposition 1. Let $G$ be a quaternionic real form, a small system of positive roots $\Psi, \mathfrak{s u}_{2}(\beta), \mathfrak{k}_{2}, H$ as in the previous section. Let $\Sigma$ be a system of positive roots in $\Phi(\mathfrak{g}, \mathfrak{t})$ so that $\Delta \subset \Sigma$. Then, a square integrable irreducible representation with Harish-Chandra parameter dominant with respect to $\Sigma$ has an admissible restriction to $H$ if and only if $\Sigma=\Psi$.

Proof. From the list of Vogan diagrams, we notice that there exists a subgroup of $H_{1}$ of $G$ so that $\left(G, H_{1}\right)$ is a symmetric pair and $H \subset H_{1}$ and $T \subset H_{1}$. Hence, if $\Sigma \supset \Delta$ is a system of positive roots for $\Phi(\mathfrak{g}, \mathfrak{t})$ so that some irreducible square integrable representation $\pi_{\mu}^{G}$ with $\mu$ dominant with respect to $\sigma$ has admissible restriction to $H$, then [6. Theorem 2.8] implies that $\pi_{\mu}^{G}$ has admissible restriction $H_{1}$. Owing to [1, Prop. 2], we have that $\mathfrak{k}_{1}(\Sigma)$ is a subalgebra of $\mathfrak{h}_{1}$. Except for some $G$ locally isomorphic to $\mathrm{SO}(4,2 n)$, the Lie algebra $\mathfrak{k}$ is the sum of two simple ideals, hence $\mathfrak{k}_{1}(\Sigma)$ is equal to $\mathfrak{s u}_{2}(\beta)$. A case by case computation forces $\Sigma=\Psi$. For a group $G$ locally isomorphic to $\mathrm{SO}(4,2 n), n \geq 2$, we select two different choices of $H_{1}$ which forces once again $\mathfrak{k}_{1}(\Sigma)$ to be equal to a copy of $\mathfrak{s u}_{2}(\beta)$ and $\Sigma=\Psi$.

\section{OTHER GROUPS}

A group $G$ locally isomorphic to either $\mathrm{SO}(3, n)$ or $\operatorname{Sp}(1, n)$ shares with the quaternionic real forms that a copy $\mathfrak{s u}_{2}$ is an ideal in any maximal compactly embedded subalgebra of $\mathfrak{g}$. Next, we analyze admissible restrictions of square integrable representations to the subgroup corresponding to the copy of $\mathfrak{s u}_{2}$ mentioned in the previous sentence.

We recall that from a criterion of Harish-Chandra it follows that a group locally isomorphic to $\mathrm{SO}(3,2 n+1)$ has no irreducible square integrable representation, whereas a group locally isomorphic to $\mathrm{SO}(3,2 n)$ does have a non empty discrete series. For a group $G$ locally isomorphic to $\mathrm{SO}(3, p)$ a maximally compactly imbedded subalgebra is isomorphic to the direct sum of the ideals $\mathfrak{s o}(3), \mathfrak{s o}(p)$. For the next statement we denote the analytic subgroup of $G$ corresponding to $\mathfrak{s o}(3)$ by $K_{1}$.

Proposition 2. For a group $G$ locally isomorphic to $\mathrm{SO}(3,2 n)$ no irreducible square integrable representation has an admissible restriction to $K_{1}$.

Proof. Because $n \geq 1$ we have that $K_{1}$ is contained in a subgroup $H_{1}$ of $G$ locally isomorphic to $\mathrm{SO}(3,1)$. Next, we recall Theorem 1.2 in [5] which gives us: If a unitary representation of $G$ has an admissible restriction to $K_{1}$ then it has an admissible restriction to $H_{1}$. Hence, if an irreducible square integrable representation of $G$ had admissible restriction to $K_{1}$ we would have that $H_{1}$ has a nonempty discrete series, which is not true since $H_{1}$ is locally isomorphic to $\mathrm{SO}(3,1)$. Another proof follows from [1] and the fact that $K_{1}(\Psi)$ is never equal to $K_{1}$. 
For a group $G$ locally isomorphic to $\operatorname{Sp}(1, q)$ we fix a maximal compact subgroup $K$ and a compact Cartan subgroup $T$. Therefore, there exists an orthogonal basis $\left\{\epsilon_{1}, \delta_{1}, \ldots, \delta_{q}\right\}$ for $i \mathrm{t}^{\star}$ and a system of positive roots $\Sigma$ so that

$$
\Sigma \cap \Phi_{c}=\left\{2 \epsilon_{1}, \delta_{i} \pm \delta_{j}, 1 \leq i<j \leq q, 2 \delta_{j}, j=1, \ldots, q\right\}
$$

and $\Sigma \cap \Phi_{n}=\left\{\epsilon_{1} \pm \delta_{j}, j=1, \ldots, q\right\}$. The simple roots are $\epsilon_{1}-\delta_{1}, \delta_{j}-\delta_{j+1}, j=$ $1, \ldots, q, 2 \delta_{q}$. The maximal root is $\beta=2 \epsilon_{1}$. It readily follows that $\mathfrak{k}_{1}(\Sigma)=\mathfrak{s u}_{2}\left(2 \epsilon_{1}\right)$. Let $\mathfrak{h}_{0}$ denote the real form of the Lie subalgebra spanned by the root vectors corresponding to the roots

$$
\Phi\left(\mathfrak{h}_{0}, \mathfrak{u}\right):=\left\{ \pm 2 \epsilon_{1}, \pm 2 \delta_{1}, \pm\left(\epsilon_{1} \pm \delta_{1}\right)\right\} .
$$

Then, $\mathfrak{h}_{0}$ is isomorphic to $\mathfrak{s p}(1,1)$. As for the quaternionic case, let $H_{0}$ denote the analytic subgroup of $G$ associated to $\mathfrak{h}_{0}$. Owing to [1, Theorem 1], we have that for $\lambda$ dominant with respect to $\Sigma$ the representation $\pi_{\lambda}^{G}$ restricted to $H_{0}$ is admissible. Let $\mu_{j}, n^{G, H_{0}}\left(\lambda, \mu_{j}\right)$ be as in (1.1). Let $\Sigma_{q}:=\Sigma \cap \Phi\left(\mathfrak{h}_{0}, \mathfrak{u}\right)$. Let $H C_{L \cap K_{2}}\left(\pi_{\lambda_{2}}^{K_{2}}\right)$ denote the set of Harish-Chandra parameters for the $L \cap K_{2}$-irreducible factors of the restriction of $\pi_{\lambda_{2}}^{K_{2}}$ to the subgroup $L \cap K_{2}$. We have:

Proposition 3. Assume $\lambda$ is dominant with respect to $\Sigma$. Then, for $j=1, \ldots$ the parameters $\mu_{j}:=\lambda_{1}+\sigma+j \epsilon_{1}$ are dominant with respect to $\Sigma_{q}$. Besides, $n^{G, H_{0}}(\lambda, \mu) \neq 0$ if and only if $\mu=\mu_{j}$ for some $j$. Moreover,

$$
n^{G, H_{0}}(\lambda, \mu)=\sum_{\substack{\sigma \in H C_{L \cap K_{2}}\left(\pi_{\lambda_{2}}^{K_{2}}\right), p \in \mathbb{Z}_{\geq 0} \\
\mu=\lambda_{1}+\sigma+p \epsilon_{1}}} M\left(\lambda_{2}, \sigma\right)\left(\begin{array}{c}
p+2 q-3 \\
2 q-3
\end{array}\right) .
$$

Proof. We begin writing the equalities (2.4) and (2.5) for the setting of the Proposition. For this particular case $(2.4)$ reads

$$
\begin{aligned}
& \sum_{\mu \in H C_{L \cap K_{2}}\left(\pi_{\lambda_{2}}^{K_{2}}\right)} M\left(\lambda_{2}, \mu\right) \sum_{r \in W\left(L \cap K_{2}, U \cap K_{2}\right)} \epsilon(r) \delta_{r \mu} \\
& =\sum_{s \in W_{\mathfrak{\jmath}} \backslash W\left(\mathfrak{k}_{2}, \mathfrak{t}\right)} \epsilon(s) \varpi\left(s \lambda_{2}\right) \delta_{q_{\mathfrak{u} \cap \mathfrak{k}_{2}}}\left(s \lambda_{2}\right) \star y_{\Delta\left(\mathfrak{k}_{2} / \mathfrak{r} \cap \mathfrak{k}_{2}\right)} .
\end{aligned}
$$

The multiset

$$
S_{w}^{H_{0}}:=\left[\Delta(\mathfrak{k} / \mathfrak{l}) \cup q_{\mathfrak{u}}\left(w \Psi_{n}\right)\right] \backslash \Phi(\mathfrak{h}, \mathfrak{u})
$$

is strict. This follows from an explicit computation of $S_{w}^{H_{0}}$, which we will carry out after the next formula. The formula (2.5) becomes:

$$
\begin{gathered}
\sum_{\substack{\mu \in \mathfrak{u}^{\star} \\
\left(\mu, \epsilon_{1}\right)>0,\left(\mu, \delta_{1}\right)>0}} n^{G, H}(\lambda, \mu)\left(\sum_{t \in W(L, U)} \epsilon(t) \delta_{t \mu}\right) \\
=\sum_{w \in W_{\mathfrak{\jmath}} \backslash W} \epsilon_{1}(w) \varpi(w \lambda) \delta_{q_{\mathfrak{u}}(w \lambda) \star y_{S_{w}^{H}} .}
\end{gathered}
$$


In this case, $\mathfrak{u} \cap \mathfrak{k}_{1}=\mathbb{R} i \check{\epsilon}_{1}, \mathfrak{u} \cap \mathfrak{k}_{2}=\mathbb{R} i \check{\delta}_{1}$, and $\mathfrak{l} \cap \mathfrak{k}_{2}=\mathfrak{s u}_{2}\left(2 \delta_{1}\right)$. Furthermore, $\Psi \cap \Phi_{\mathfrak{z}}=\left\{\delta_{i} \pm \delta_{j}, 2 \leq i<j \leq q\right\}, W=\left\langle S_{2 \epsilon_{1}}\right\rangle \times W\left(\mathfrak{k}_{2}, \mathfrak{t}_{2}\right)$, and $W_{\mathfrak{z}} \backslash W=$ $\left\langle S_{2 \epsilon_{1}}\right\rangle \times W_{\mathfrak{z}} \backslash W\left(\mathfrak{k}_{2}, \mathfrak{t}_{2}\right)$.

For $w \in W\left(\mathfrak{k}_{2}, \mathfrak{t}_{2}\right), w \Psi_{n}=\Psi_{n}, w S_{2 \epsilon_{1}} \Psi_{n}=-\Psi_{n}$ and

$$
\begin{aligned}
q_{\mathfrak{u}}\left(w \Psi_{n}\right) \backslash \Phi(\mathfrak{h}, \mathfrak{u}) & =\underbrace{\left\{\epsilon_{1}, \ldots, \epsilon_{1}\right\}}_{2(q-1)} \\
q_{\mathfrak{u}}\left(w S_{2 \epsilon_{1}} \Psi_{n}\right) \backslash \Phi(\mathfrak{h}, \mathfrak{u}) & =\underbrace{\left\{-\epsilon_{1}, \ldots,-\epsilon_{2}\right\}}_{2(q-1)} \\
\Delta(\mathfrak{k}, \mathfrak{l})=q_{\mathfrak{u}}\left(\Psi_{c} \backslash \Phi_{\mathfrak{z}}\right) \backslash \Phi(\mathfrak{h}, \mathfrak{u}) & =\{2 \epsilon_{1}, 2 \delta_{1}, \underbrace{\delta_{1}, \ldots, \delta_{1}}_{2(q-1)}\} \backslash \Phi(\mathfrak{h}, \mathfrak{u})=\Delta\left(\mathfrak{k}_{2}, \mathfrak{l} \cap \mathfrak{k}_{2}\right) .
\end{aligned}
$$

Therefore, for $w \in W\left(\mathfrak{k}_{2}, \mathfrak{t}\right)$ we have

$$
\begin{aligned}
S_{w}^{H_{0}} & =\underbrace{\left\{\epsilon_{1}, \ldots, \epsilon_{1}\right\}}_{2(q-1)} \cup \Delta\left(\mathfrak{k}_{2}, \mathfrak{l} \cap \mathfrak{k}_{2}\right) \\
S_{S_{2} \epsilon_{1} w}^{H_{0}} & =\underbrace{\left\{\epsilon_{1}, \ldots, \epsilon_{1}\right\}}_{2(q-1)} \cup \Delta\left(\mathfrak{k}_{2}, \mathfrak{l} \cap \mathfrak{k}_{2}\right) .
\end{aligned}
$$

After replacing $S_{w}^{H_{0}}$ by the result obtained in the previous line, the right hand side of the formula similar to the one in (2.5) becomes

$$
\begin{aligned}
\sum_{\substack{t \in\left\{1, S_{\epsilon_{1}}\right\} \\
s \in W_{\mathfrak{z}} \backslash W\left(\mathfrak{k}_{2}, \mathfrak{t}_{2}\right)}} \epsilon(t) \epsilon(s) \varpi\left(s \lambda_{2}\right) \delta_{t \lambda_{1}} \star \delta_{q_{\mathfrak{u}}\left(s \lambda_{2}\right)} \star y_{\Delta\left(\mathfrak{k}_{2} / \mathfrak{r} \cap \mathfrak{k}_{2}\right)} \star y_{t \epsilon_{1}}^{2(q-1)} \\
=\sum_{t \in\left\{1, S_{2 \epsilon_{1}}\right\}} \epsilon(t) \sum_{\substack{r \in W\left(\mathfrak{k}_{2}, \mathfrak{t}_{2}\right) \\
\sigma \in H C}} \epsilon(r) M\left(\lambda_{2}, \sigma\right) \delta_{t \lambda_{1}+r \sigma} \star y_{t \epsilon_{1}}^{2(q-1)} \\
=\sum_{\substack{t, r, \sigma \\
p \in \mathbb{Z} \geq 0}} \epsilon(t) \epsilon(s) M\left(\lambda_{2}, \sigma\right)\left(\begin{array}{c}
p+2(q-1)-1 \\
2(q-1)-1
\end{array}\right) \delta_{t r\left(\lambda_{1}+\sigma+p \epsilon_{1}\right)} \\
=\sum_{p \geq 0} \sum_{\sigma} M\left(\lambda_{2}, \sigma\right)\left(\begin{array}{c}
p+2 q-3 \\
2 q-3
\end{array}\right) \sum_{w \in W(L, U)} \epsilon(w) \delta_{w\left(\lambda_{1}+\sigma+p \epsilon_{1}\right)} .
\end{aligned}
$$

By a reasoning similar to (2.7) we obtain that $\lambda_{1}+\sigma+p \epsilon_{1}$ is dominant with respect to $\Psi_{q}$ and we have concluded the proof of Proposition 3 .

\section{SIMONDI'S THESIS}

Sebastián Simondi's Ph.D. thesis [9] was defended in March 2007. Most of his results were verified in a case by case checking. By now, some of his results are a consequence of work of Toshi Kobayashi [7, 6, 1]. This section does not include proofs of the stated results; we will point out those results that follow from the 
work of T. Kobayashi, Kobayashi-Oshima, and Duflo-Vargas, and those results we believe are still not in the literature.

We now describe the results. For this, we fix a noncompact connected matrix simple Lie group $G$ and a maximal compact subgroup $K$ for $G$. Henceforth, $H$ is a closed reductive subgroup of $G$ so that $L:=H \cap K$ is a maximal compact subgroup for $H$ and that $(G, H)$ is a symmetric pair. Hence, $(K, L)$ is a Riemannian symmetric pair.

As in the previous sections we assume $G$ admits irreducible square integrable representations. We would like to point out that in the course of the computation extensive use was made of the description for the set of equivalence classes of square integrable irreducible representations given by Harish-Chandra in terms of Harish-Chandra parameters.

In [1, 5, 7, 6] we find criteria for checking whether or not the restriction of an square integrable representation for $G$ is an admissible representation for a subgroup $H$. By means of these criteria, the classification of the symmetric pairs given by Berger and a case by case checking, we have:

Theorem 2. Assume $(G, H)$ is a symmetric pair and $(\pi, V)$ is an irreducible square integrable representation for $G$. If $\mathfrak{k}$ is a simple Lie algebra, then the restriction of $\pi$ to $H$ is not an admissible representation.

Nowadays, this result follows from [1] or from [8].

For the next result we fix a maximal compact connected subgroup $L^{\prime}$ for $K$ so that the rank of $K$ is equal to the rank of $L^{\prime}$.

Theorem 3. Let $(\pi, V)$ be an irreducible square integrable representation for $G$. We assume $\mathfrak{k}$ is a simple Lie algebra. Then, $\pi$ restricted to $L^{\prime}$ is not an admissible representation.

When $L^{\prime}$ is a maximal compact subgroup of a reductive subgroup $H$ of $G$ so that $(G, H)$ is a symmetric pair, Theorem 3 follows from Theorem 2 and results in [1]. For the other subgroups $L^{\prime}$ the proof has been done in a case by case checking based on the classification of the equal rank maximal subgroups of $K$ obtained by Borel-de Siebenthal and work of Toshi Kobayashi on criteria on admissibility of restriction of representations.

Under the hypothesis that $\mathfrak{k}$ is not a simple Lie algebra, $(G, H)$ a symmetric pair, and the subgroups $L, K$ are of the same rank, we obtain a complete list, in the language of Harish-Chandra parameters, of the square integrable representations for $G$ which do not have an admissible restriction to $H$. Nowadays these results are included in [8, 11].

For the last result of this note we further assume $(G, K)$ is an Hermitian symmetric pair. Then, the center of $K$ is a one dimensional torus. Let $K_{s s}$ denote the semisimple factor of $K$. We fix a maximal torus $T$ for $K$. The hypothesis on $(G, K)$ allows us to choose, once for all, a holomorphic system of positive roots $\Psi_{h}$ in $\Phi(\mathfrak{g}, \mathfrak{t})$. In [5] it is shown that either a holomorphic or a antiholomorphic discrete series for $G$ has an admissible restriction to $K_{s s}$ if and only if $G / K$ is not 
a tube domain. The next result gives a criterion which allows to determine when an arbitrary irreducible square integrable representation has admissible restriction to $K_{s s}$. For this we recall that the set of equivalence classes for irreducible square integrable representations is parameterized by the set of Harish-Chandra parameters $\lambda$ dominant with respect to $\Delta:=\Psi_{h} \cap \Phi_{c}$. For a Harish-Chandra parameter $\lambda$ dominant with respect to $\Delta$, the regularity of $\lambda$ determines a system of positive $\operatorname{roots} \Psi_{\lambda}:=\{\alpha \in \Phi(\mathfrak{g}, \mathfrak{t}): \lambda(\check{\alpha})>0\}$ which satisfies $\Psi_{\lambda} \cap \Phi_{c}=\Delta$. In the table below, we list for each Hermitian symmetric pair $(G, K)$ subsets $I, \tilde{I}$ of $\Psi_{h}$.

\begin{tabular}{|c|c|c|}
\hline $\mathfrak{g}$ & $\mathfrak{k}$ & \\
\hline $\mathfrak{s p}(n, \mathbb{R})$ & $\mathfrak{u}(n)$ & $\begin{array}{l}\text { If } \begin{aligned} n & =2 l \\
$\[ \]$ & =\left\{\left(e_{k}+e_{n-k+1}\right)\right\}_{k=1}^{l} \\
\tilde{I} & =-I \\
\text { If } n & =2 l+1, \\
I & =\left\{2 e_{l+1}\right\} \cup\left\{\left(e_{k}+e_{n-k+1}\right)\right\}_{k=1}^{l} \\
\tilde{I} & =-I\end{aligned}\end{array}$ \\
\hline $\mathfrak{s o}^{*}(2 n)$ & $\mathfrak{u}(n)$ & 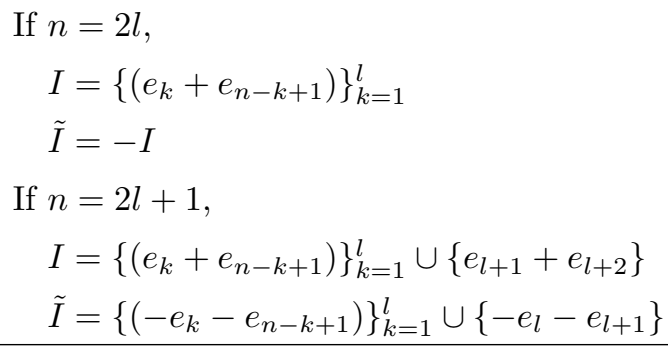 \\
\hline $\mathfrak{s u}(p, q)$ & $\mathfrak{s u}(p) \oplus \mathfrak{u}(q)$ & $\begin{array}{l}I=\left\{e_{i}-e_{\gamma_{i}}\right\}_{i=1}^{p} \\
\tilde{I}=\left\{-e_{i}+e_{b_{i}}\right\}_{i=1}^{p}\end{array}$ \\
\hline $\mathfrak{e}_{6(-14)}$ & $\mathfrak{s o}(10) \oplus \mathfrak{s o}(2)$ & $\begin{array}{l}I=\left\{\varepsilon_{1}, \varepsilon_{2}, e_{1}+e_{5}, e_{2}+e_{5}\right\} \\
\tilde{I}=-I\end{array}$ \\
\hline $\mathfrak{e}_{7(-25)}$ & $\mathfrak{e}_{6} \oplus \mathfrak{s o}(2)$ & $\begin{array}{l}I=\left\{\eta_{1}, \eta_{2}, e_{1}+e_{6}\right\} \\
\tilde{I}=-I\end{array}$ \\
\hline
\end{tabular}

Theorem 4. Assume $(G, K)$ is an Hermitian symmetric pair and fix an irreducible square integrable irreducible representation $\left(\pi_{\lambda}^{G}, V_{\lambda}\right)$ for $G$ of Harish-Chandra parameter $\lambda$. Without loss of generality we may and will assume $\lambda$ is dominant with respect to $\Delta$. We further assume $\pi_{\lambda}^{G}$ is neither holomorphic nor antiholomorphic. Then, $\pi_{\lambda}^{G}$ restricted to $K_{s s}$ is an admissible representation if and only if either $I$ or $\tilde{I}$ is a subset of $\Psi_{\lambda}$. 
The proof of the last theorem is carried out based on the classification of Hermitian symmetric pairs and criteria due to Kobayashi [7, 6].

Here, in notation of Bourbaki:

$$
\begin{aligned}
& \gamma_{i}=i+\left[\frac{(i-1)(q-p)}{p}\right]+p, \quad \text { for } 1 \leq i \leq p, \\
& b_{i}=\left\{\begin{array}{ll}
i+1+\left[\frac{i(q-p)}{p}\right]+p & \text { if } \frac{i(q-p)}{p} \notin \mathbb{Z}, \\
i+\frac{i(q-p)}{p}+p & \text { if } \frac{i(q-p)}{p} \in \mathbb{Z},
\end{array} \text { for } 1 \leq i \leq p,\right. \\
& \varepsilon_{1}= \frac{1}{2}\left(-e_{1}-e_{2}-e_{3}-e_{4}+e_{5}-e_{6}-e_{7}+e_{8}\right), \\
& \varepsilon_{2}=\frac{1}{2}\left(-e_{1}-e_{2}+e_{3}+e_{4}+e_{5}-e_{6}-e_{7}+e_{8}\right), \\
& \eta_{1}=\frac{1}{2}\left(-e_{1}+e_{2}-e_{3}-e_{4}+e_{5}+e_{6}-e_{7}+e_{8}\right), \\
& \eta_{2}=\frac{1}{2}\left(-e_{1}-e_{2}+e_{3}+e_{4}-e_{5}+e_{6}-e_{7}+e_{8}\right) .
\end{aligned}
$$

\section{REFERENCES}

[1] Duflo, M., Vargas, J., Branching laws for square integrable representations, Proc. Japan Acad. Ser. A Math. Sci. 86 (2010), no. 3, 49-54. MR 2641797

[2] Gross, B., Wallach, N., On quaternionic discrete series representations, and their continuations, J. Reine Angew. Math. 481 (1996), 73-123. MR 1421947

[3] Hecht, H., Schmid, W., A proof of Blattner's conjecture, Invent. Math. 31 (1975), no. 2, 129-154. MR 0396855

[4] Heckman, G. J., Projections of orbits and asymptotic behavior of multiplicities for compact connected Lie groups, Invent. Math. 67 (1982), no. 2, 333-356. MR 0665160

[5] Kobayashi, T., Discrete decomposability of the restriction of $A_{\mathfrak{q}}(\lambda)$ with respect to reductive subgroups and its applications, Invent. Math. 117 (1994), no. 2, 181-205. MR 1273263

[6] Kobayashi, T., Discrete decomposability of the restriction of $A_{\mathfrak{q}}(\lambda)$ with respect to reductive subgroups. II. Micro-local analysis and asymptotic $K$-support, Ann. of Math. (2) 147 (1998), no. 3, 709-729. MR 1637667.

[7] Kobayashi, T., Discrete decomposability of the restriction of $A_{\mathfrak{q}}(\lambda)$ with respect to reductive subgroups. III. Restriction of Harish-Chandra modules and associated varieties, Invent. Math. 131 (1998), no. 2, 229-256. MR 1608642

[8] Kobayashi, T., Oshima, Y., Classification of discretely decomposable $A_{\mathfrak{q}}(\lambda)$ with respect to reductive symmetric pairs. Adv. Math. 231 (2012), no. 3-4, 2013-2047. MR 2964632

[9] Simondi, Sebastián, Restricción de representaciones de cuadrado integrable. Ph.D. thesis, Universidad Nacional de Córdoba, 2007. http://www2.famaf.unc.edu.ar/publicaciones/ documents/serie_d/DMat49.pdf

[10] Vargas, J., Harish Chandra modules of rank one Lie groups with admissible restriction to some reductive subgroup, J. Lie Theory 20 (2010), no. 4, 643-663. MR 2778229

[11] Vargas, J., Associated symmetric pair and multiplicities of admissible restriction of discrete series, Internat. J. Math. 27 (2016), no. 12, 1650100. MR 3575924 
[12] Wallach, N., Real reductive groups I, Academic Press, Boston, 1988. MR 0929683

[13] Wallach, N., Generalized Whittaker vectors for holomorphic and quaternionic representations, Comment. Math. Helv. 78 (2003), no. 2, 266-307. MR 1988198.

O. Márquez

Universidade Federal de Santa Maria, Santa Maria - RS, Brasil

omskar@gmail.com

S. Simondi

Facultad de Ciencias Exactas y Naturales, Universidad Nacional de Cuyo, 5500 Mendoza, Argentina

ssimondi@uncu. edu. ar

J. A. Vargas ${ }^{凶}$

FAMAF-CIEM, Ciudad Universitaria, 5000 Córdoba, Argentina

vargas@famaf .unc.edu.ar

Received: December 5, 2017

Accepted: July 9, 2018 